Appears in R. Chin and T.C. Pong (eds), Lecture Notes in Computer Science

Vol. 1351, Springer-Verlag, 1997; also appears in the

Proceedings of the 3rd Asian Conference on Computer Vision,

Hong Kong, January 1998.

\title{
Detecting Targets in SAR Images: A Machine Learning Approach
}

\author{
Qi Zhang Zoran Duric Ryszard S. Michalski* \\ George Mason University \\ 4400 University Dr., Fairfax, VA 22030, USA \\ *Also with GMU Departments of Computer Science and Systems \\ Engineering, and the Institute of Computer Sciences, Polish Academy of Science
}

\begin{abstract}
This paper describes a novel application of the MIST methodology to target detection in SAR images. Specifically, a polarimetric whitening filter and a constant false alarm rate detector are used to preprocess a SAR image; then the AQ15c learning program is applied to learn and detect targets. Encouraging and impressive experimental results are provided.
\end{abstract}

KEYWORD: Learning in vision, target detection in SAR images.

\section{Introduction}

Research on computer vision reveals that it is necessary for a flexible and robust vision system to incorporate learning capabilities. Now this line of research has become an active area (Michalski et al., 1992; Bhanu \& Poggo, 1994). This paper is focused on using machine learning methods to detect targets in SAR images.

A synthetic aperture radar (SAR) is an all-weather imaging device, able to provide good images of what it has detected even in fog, clouds, or darkness in which optical sensors are useless. Detecting and recognizing targets in SAR images is of much significance in military and civil applications. Successful detection of targets in SAR images is difficult because there is a large amount of noise in image data. This paper presents a novel machine learning approach to this problem by using the MIST/AQ method (Michalski et al., 1996). In particular, a SAR image is first processed by a polarimetric whitening filter (PWF) to improve image quality (Novak et al., 1990), and then input to a constant false alarm rate (CFAR) detector for screening its natural clutter and detecting potential targets within it (Ravid \& Levanon, 1992). The output from the CFAR detector is used by AQ15c (Michalski. et. al., 1986; Wnek et. al., 1996) for training and detection. The $1 \mathrm{ft} \times 1 \mathrm{ft}$ resolution SAR images we used were collected in Stockbridge New York, by the Lincoln Laboratory at MIT and provided to us by DARPA. The experimental results were very encouraging and impressive.

\section{Related Work}

Target detection in SAR images is difficult because of large amount of noise in image data. Kreithen et al. (1993) used the polarimetric whitening filter and a CFAR detector to preprocess SAR images. After grouping sets of clustered pixels (potential targets) which were seemingly from the same targets, attribute values were generated for each of them and a quadratic distance for each potential target was calculated and compared to a threshold determined previously by experiments. Obviously, a single 
threshold is not so understandable or flexible as symbolic knowledge descriptions. Besides, determining which pixels came from a target or not and then grouping them is often difficult or impossible due to noise. Burl et al. (1994) used the matched filter technique to detect potential volcanoes in Venus SAR images. Matched filters for each kind of volcano were constructed from training volcano examples and then were applied to scan an image pixel by pixel to locate potential volcanoes. The matched filter is possibly subject to rotation, size and other vision condition changes. Further, using matched filter to scan a whole image and computing the degrees of match with each constructed filter is time-consuming.

Application of machine learning techniques to target detection in images is relatively new. Rong and Bhanu (1996) adopted the reinforcement learning method. The training and testing were directly performed on raw FLIR (forward-looking infrared radar, not SAR) images without any transformation. They divided an image into many rectangulars which were the input unit to a learning system. Directly training and testing on raw data could consume more time and generate harder learning problems and dividing the image into small areas for training could lead to incompleteness of target information.

\section{MIST Methodology}

Among the most important research goals of applying machine learning methods to vision problems is to gain better understanding of matching appropriate learning methods to appropriate vision problems. MIST (Multilevel Image Sampling and Transformation) has been developed as a general methodology for applying machine learning methods to vision problems (Michalski et al., 1996). The purpose of MIST is to provide a researcher with an environment in which a variety of machine learning methods and approaches can be flexibly applied to a wide range of vision problems.

The MIST methodology works in two modes: training mode and interpretation mode (Figure 1). In the training mode, four steps are needed and, based on training performance, possibly repeated for better results. Event generation is to generate examples for learning or testing. Description space generation means that a trainer assigns concept names to areas in training images that contain concepts or objects to be learned. In the interpretation mode, three steps are executed. Transformation application is to apply the Image Knowledge Base (IKB) to examples generated from testing images to produce a transformed version which, for instance, could be a segmented image. The output is annotated symbolic images (ASI). In an ASI, areas or objects that correspond to the recognized concepts in the original image are marked by symbols (e.g., colors) denoting these concepts and linked to concept annotations (text containing additional information about that concept, such as degree of certainty of recognition, properties of the concepts, relation to other concepts, etc.). The output ASI in one level can be the end result (one-level training) or input to later levels (multilevel training) for better results (e.g., repeated training on the same natural scene image) or for other tasks (e.g., target recognition). The image knowledge base (IKB) contains prior or learned descriptions or visual concepts, image processing operators, attribute extraction operators and background knowledge relevant to image interpretation. 
Among the advantages of this methodology are the ease of applying and testing diverse learning methods in a uniform manner, the potential for implementing advanced and complex learning processes, the natural way of interpreting images.

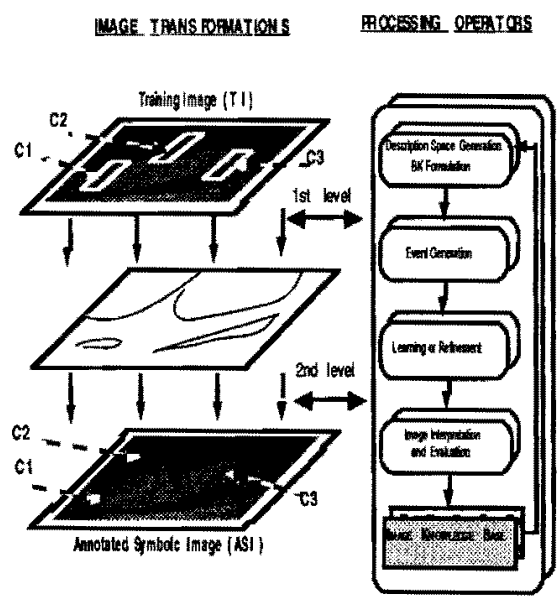

(a)

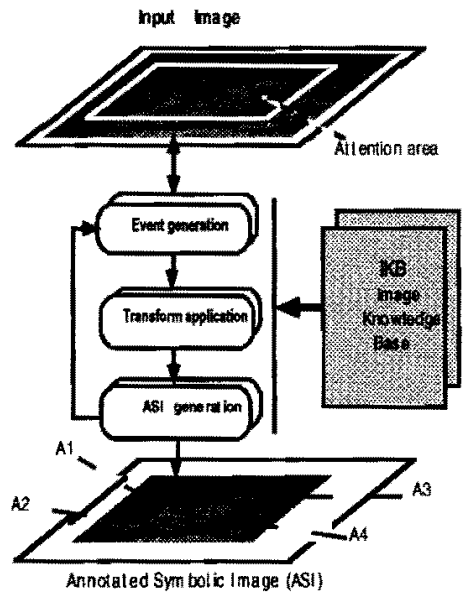

(b)

Fig. 1. The MIST methodology (a) training mode, (b) interpretation mode.

\section{Learning to Detect Targets}

Guided by the MIST methodology, we designed a MIST/AQ system which consists of three sequential phases: enhancing image quality by a PWF, screening by a CFAR detector, and target learning and detection by AQ15c (Figure 2). The first two stages provide the preprocessing needed for further target detection and recognition.

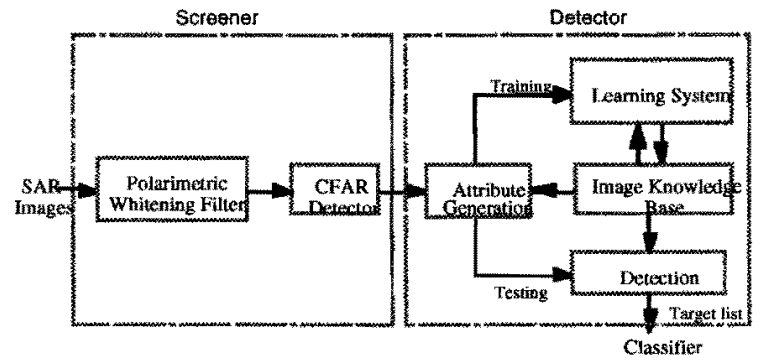

Fig. 2. The system architecture.

\subsection{Polarimetric Whitening Filter}

The technique called the polarimetric whitening filter (PWF) (Novak et al,, 1990) improves SAR image quality in two aspects: minimization of the amount of speckle (noise) and sharpening the edges of image objects. For the details of this technique, see Novak et al.(1990) and for its application to this work, see Zhang et al. (1996).

\subsection{CFAR Screening}

Various constant false rate alarm (CFAR) algorithms (e.g., Ravad \& Levanon, 1992; Wang et al., 1994) take a SAR image as input and perform a screening function, i.e., 
detecting potential targets in SAR images by examining intensities of radar returns and thereby providing a massive reduction of natural clutter (grass, trees etc.). Figure $3 b$ is the CFAR image (i.e., processed by a CFAR detector) of Figure 3a. In our work, we followed the implementation presented in (Wang et al., 1994). Note that due to noise inherent in SAR images, there are many false alarms which passed the screening of the CFAR detector.

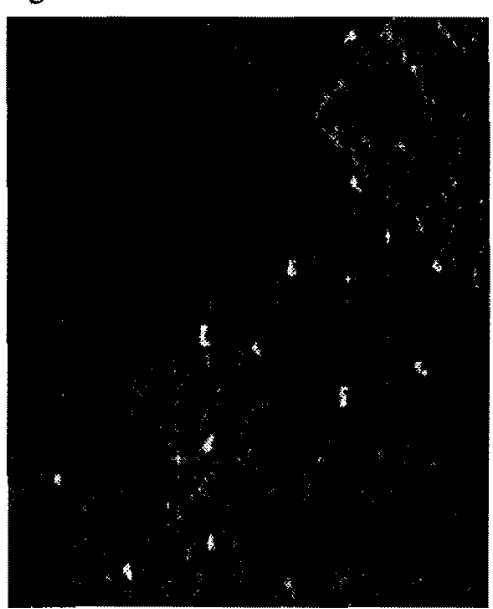

(a)

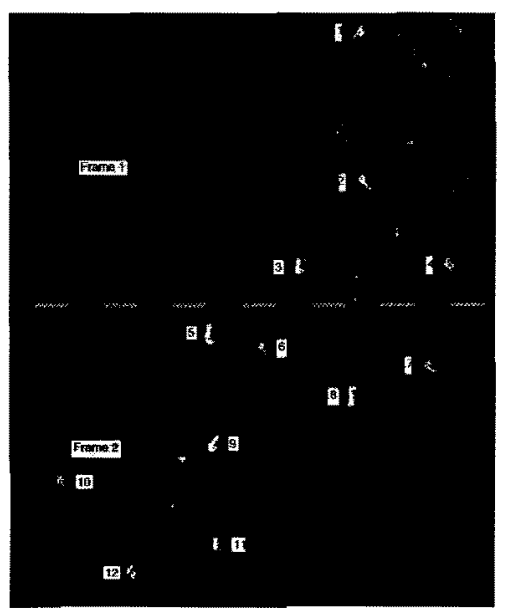

(b)

Fig. 3. An exemplary (a) PWF image and (b) CFAR image containing targets: 2,11: M60-tank; 3,7,9: M48-tank; 4,10: M84-APC; 6: M113-APC; 12: M59-APC; 5,8: M55-howitzer; 1: unknown.

\subsection{Learning and Detecting}

\subsubsection{Definitions of attributes}

In our work, the pixel is the unit used in learning and detection. We consider each pixel that passed the CFAR detector as an example. For each example, produce a set of attribute values defined on a circular area (centered on the pixel or example of interest) from either its CFAR or PWF image, both of which can provide descriptive information about the example (Figure 4a). Two circular areas were used in this work, one with the diameter being 21 pixels and the other 31 pixels. For the definitions of adopted attributes, see Zhang et al. (1996).

\subsubsection{Classification of training examples and discretization}

Before learning, training examples must be classified as target and non-target examples. This is not easy, since it is impossible to accurately decide whether the pixels on the border of a target are target examples or non-target examples. Thus, there might be some misclassified examples for training. We adopted a simple rule: pixels connected (8-connectivity) to a target are target examples and otherwise nontarget examples (Figure 4b).

Considering the characteristics of SAR image data, we adopted the Chi-merge discretization scheme (Kerber, 1992) and applied it to extracted raw data from CFAR or PWF images. The following are exemplary examples after discretization 
(attributes ending in "1" represent attributes defined on a circle with ta diameter of 21 pixels, those ending in " 2 " are for circles with a 31-pixel diameter):

Target examples

(1) $[$ power $1=49] \quad[\mathrm{sd} 1=24] \quad[$ fractal1 $=7] \quad[$ areal $=3] \quad[$ wrfr $1=8] \quad[$ power $2=25] \quad[s d 2=10]$ [fractal2 $=6][$ area2 $=3]$ [wrfr $2=4]$

(2) $[$ power1 $=47][\operatorname{sd} 1=24] \quad[$ fractal1 $=7] \quad[\operatorname{area} 1=2] \quad[w r f r 1=9] \quad[$ power2 $=24] \quad[s d 2=10]$ $[$ fractal2 $=7][$ area2 $=3][w r f r 2=5]$

Non-target examples

(1) $[$ power $1=6][\mathrm{sd} 1=0][$ fractall $=0][$ area $1=0][\mathrm{wrfr} 1=0][$ power $2=0][\mathrm{sd} 2=0][$ fractal2 $=0]$ [area $2=0][$ wrfr $2=0]$

(2) $[$ power $1=0][\mathrm{sd} 1=0][$ fractall $=0][$ area $1=0][\mathrm{wrfr} 1=2][$ power $2=0][\mathrm{sd} 2=0][$ fractal2 $=0]$ [area2 $=0][$ wrfr $2=0]$

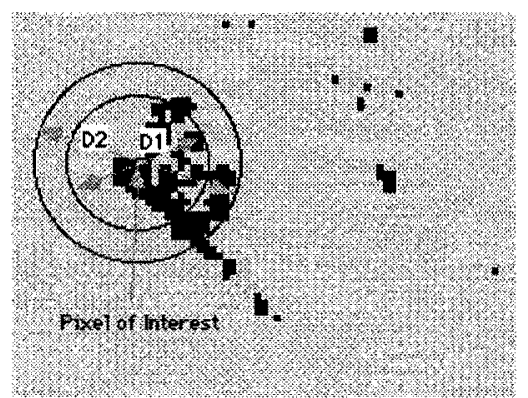

(a)

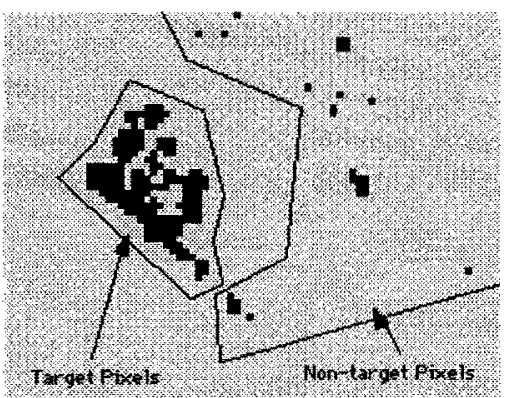

(b)

Fig. 4. Enlarged target 4 in Fig 3b. (a) Attributes of a pixel are defined on circular areas; (b) Classification of target and non-target examples.

\subsubsection{Training}

The SAR image acquired during a single imaging process by an airplane or satellite is called a pass, which can be divided into smaller portions called frames. Image objects are partitioned into two classes: targets and non-targets. The following ane exemplary AQ rules acquired by taking pixels in frame 1 of Figure 4 as training examples.

\section{Target examples}

Rule1 [sdl=13..57] \& [area1 $=0 . .10] \&[w r f r 1=0.9] \&$ [power2=19..99] \& [sd2=7..23] \& [fracta12=3..15] \& [wrfr2 $=0 . .8] \quad$ (t-weight:842, u-weight:107)

Rule2 [area1 $=10 . .12] \&[$ area2 $=0 . .20] \quad(t$-weight:742, u-weight:18)

Non-target examples

Rule1 [areal $=0 . .9] \&[a r e a 2=0 . .14] \&[w r f r 2=0.3]$ ( $t$-weight:1561, u-weight:890)

Rule2 [power1 $=0$ ] (t-weight:632, u-weight:8)

The t-weight in the above rules represents the total number of examples covered by a rule and the u-weight the number of examples uniquely covered by that rule. The larger weights are, the more stronger the pattern in a learned rule represents.

\section{Experimental Results and Discussion}

We tested all our SAR images whose ground truth is known. The experimental results are summarized in Table 1. 


\begin{tabular}{|c|c|c|c|c|c|}
\hline experiment & $\begin{array}{c}\text { \# training } \\
\text { examples }\end{array}$ & $\begin{array}{c}\text { \# testing } \\
\text { examples }\end{array}$ & $\begin{array}{c}\text { \# learned AQ } \\
\text { rules }\end{array}$ & $\begin{array}{c}\text { accuracy } \\
\text { (pixel-based) }\end{array}$ & $\begin{array}{c}\text { accuracy } \\
\text { (target-based) }\end{array}$ \\
\hline 1 & 3069 & 1706 & 12 & $98 \%$ & $100 \%$ \\
\hline 2 & 9284 & 4915 & 19 & $98 \%$ & $100 \%$ \\
\hline
\end{tabular}

Table 1. Summary of experimental results.

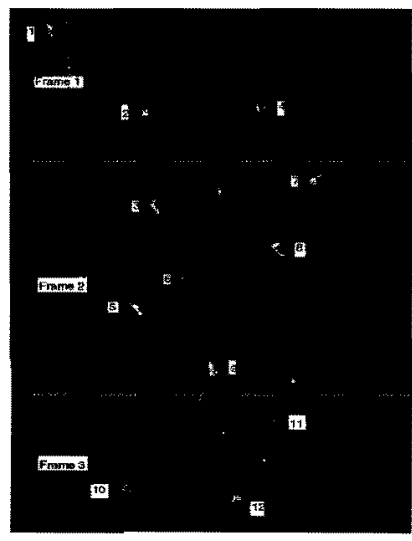

(a)

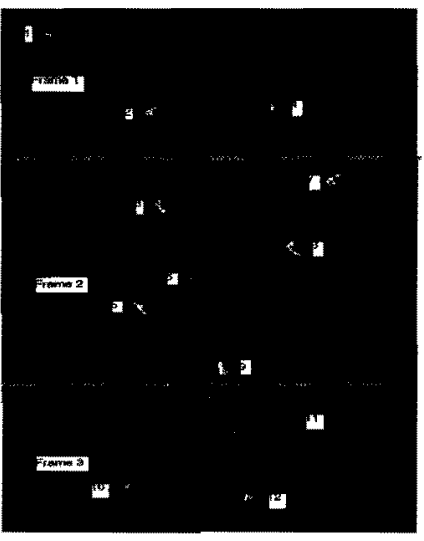

(b)

Fig. 5. Pass 7: (a) CFAR image, (b) AQ detection results. Targets are: 2,11: M60-tank; 3,7,9: M48-tank; 4,10: M84-APC; 6: M113-APC; 12: M59-APC; 5,8: M55-howitzer; 1: unknown.

In the first experiment, training was executed on Frame 1 in Figure $3 \mathrm{~b}$ and tested on Frame 2 in Figure 5a. The goal of this experiment was to see the performance of the rules learned in one pass but applied to a another place of a different pass.

In the second experiment, in addition to Frame 1 in Figure 3b, the data in Frame 2 in Figure 6a was also used for training, in which there is a power-line tower. Frame 1 in Figure $6 \mathrm{~b}$ was used for testing. As can be seen, AQ detection results were excellent (Figure 6b). Further, the rules learned in this experiment were retested in Figure $5 \mathrm{~b}$ and the results were almost the same. Note that immense number of false alarms in Figure $6 \mathrm{~b}$ indicate the difficulty in determining and grouping pixels which is the way adopted by Kreithen et al. (1993).

The first two experiments indicate that the MIST/AQ approach was able to capture the patterns among data and that learned knowledge was successfully applied to testing images, even to untrained targets. The results of the second experiment are interesting and important because it shows the necessity of learning. It can be easily seen from Figure $6 \mathrm{~b}$ that it is virtually impossible to remove non-target examples by using vision techniques such as the size filter or majority voting.

An outstanding aspect in our experiments is that all targets remained after AQ detection while false alarms were maximally reduced, almost to zero. Another thing worth mentioning is that even though there was noise in our training data the results were still satisfactory. A possible way of avoiding data noise and acquiring fewer rules is selecting only some typical target pixels (e.g., pixels in the center of a target) for learning. However rules learned in this way might not capture the various data patterns near or on the border of a target. Because of this, the spatial distribution 
(important for target recognition) of pixels of detected targets would be damaged. Further, some targets would probably disappear. Experiments proved this analysis (not shown here). In our results, the number of leamed rules was small and almost all targets were well preserved in their spatial distribution of pixels.

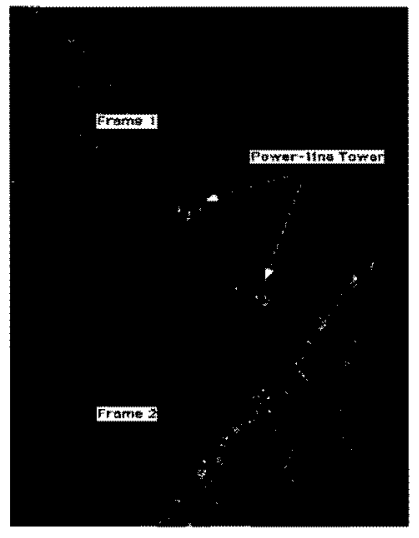

(a)

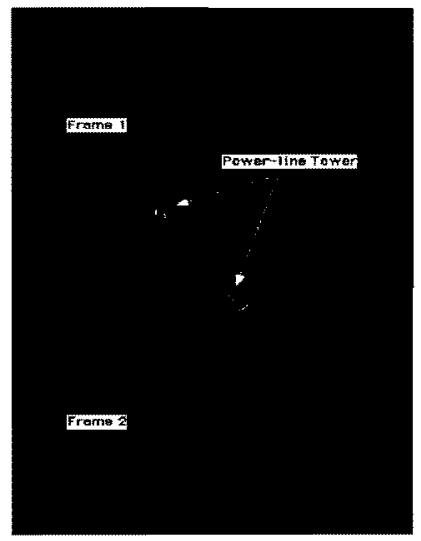

(b)

Fig. 6. Pass 5: (a) the CFAR image; (b) AQ detection results.

Our system for target detection is a good combination of vision and machine learning techniques. The noise reduction by the PWF and the screening from a CFAR detector maximally reduce and improve data needed as input to a learning method, in contrast to directly performing learning and testing on raw images (Rong \& Bhanu, 1996).

\section{Conclusion}

This paper describes a novel application of the MIST methodology to target detection in SAR images. The presented MIST/AQ system for target detection in SAR images consists of: using the polarimetric whitening filter to enhance the quality of SAR images, applying a CFAR detector to screen natural clutter to maximally reduce the unnecessary information for training, and learning and detecting targets by AQ15c. The contributions of our approach can be summarized: the pixel-based operations in our approach avoid the problem of determining and grouping pixels or building templates; utilizing vision techniques maximally reduces and cleans data necessary to learning so that learning is more likely to succeed; false alarms are maximally reduced so as to provide a classifier with a list of potential targets of good quality. Experimental results presented are very promising and clearly show the effectiveness of the MIST/AQ method for solving this problem.

\section{Acknowledgements}

We would like to thank Dr. Maloof for his help in this work, Dr. Kaufman for his technical support in preparing image data, Jim Mitchell for his comments. This research was conducted in the Machine Learning and Inference Laboratory at George Mason University. The Laboratory's activities are supported in part by the Defense Advanced Research Projects Agency under grant F49620-95-1-0462, administered by the Air Force Office of Scientific Research, in part by the National Science 
Foundation under grants DMI-9496192 and IRI-9020266, and in part by the Office of Naval Research under grant N00014-91-J-1351

\section{References}

Bhanu, B \& Poggio, T., "Introduction to the special section on learning in computer vision", IEEE Transactions on pattern analysis and machine intelligence, vol. 16, no. $9,1994$.

Burl, M.C., \& Fayyad, U.M., \& Perona, P., \& Smyth, P., \& Burl, M.P., "Automating the hunt for volcanoes on Venus", Proceedings 1994 IEEE Computer Society Conference on Computer Vision and Pattern Recognition, Seattle, WA, pp. 302-309, 1994.

Kerber, R., "ChiMerge: discretization of numeric attributes", Proceedings of the Tenth National Conference on Artificial Intelligence, San Jose, CA, pp. 123-128, 1992.

Kreithen, D.E., \& Halversen, S.D., \& Owirka, G.J., "Discriminating targets from clutter", The Lincoln Laboratory Joumal, vol. 6, no. 1, pp.25-52, 1993.

Michalski, R.S., \& Mozetic, I., \& Hong, J., \& Larvac, N., "The multipurpose incremental learning system AQ15 and its testing application to three medical domains", Proceedings of the 5th National Conference on Artificial Intelligence., 1986.

Michalski, R.S., \& Rosenfeld, A. \& Aloimonos, "Machine Vision and Learning: a report on the NSF/ARPA workshop on machine learning and vision", Harpers Ferry, WV., 1992.

Michalski, R.S., \& Zhang, Q., \& Maloof, M.A. \& Bloedorn, E., "The MIST methodology and its application to natural scene interpretation", Proceedings of Image Understanding Workshop, Palms Springs, CA., 1996.

Novak, L.M., \& Burl, M.C., Chaney, \& R.D., \& Owirka, G.J., "Optimal processing of polarimetric synthetic-aperture radar imagery", The Lincoln Laboratory Journal, vol. 3, no. 2, pp.273-290, 1990.

Ravid, \& Levanon, N., "Maximum-likelihood CFAR for Weibull background", IEE Proceedings- $F$, vol. 139, no. 3. June, 1992, pp.256-264, 1992.

Rong, S. \& Bhanu, B., "Reinforcement learning for integrating context with clutter models for target detection", Proceedings of Image Understanding Workshop, Palms Springs, CA, pp.1389-1394, 1996.

Wang, Y, \& Chellappa, R., \& Zheng, Q., "Detection of point targets in high resolution synthetic aperture radar images", Proceedings of IEEE International Conference on Acoustics, Speech and Signal Processing, vol. 5, pp9-12, 1994.

Wnek, J., \& Kaufman, K., \& Bloedorn, E., \& Michalski, R.S., "Inductive learning system AQ15c: the method and user's guide", Reports of the Machine Learning and Inference Laboratory, MLI 95-4, George Mason University, Fairfax, VA., 1995.

Zhang, Q., \& Duric, Z., Michalski, R.S., "Target detection in SAR images: the MIST/AQ method", MLI 96-28, Reports of Machine Learning and Inference Laboratory, George Mason University, Fairfax, VA., 1996. 